\title{
Analysis of Meteorological Conditions for Fog and Haze in the Eastern and Central Regions of China during January, 2013
}

\author{
Ying $\mathrm{Wu}^{1,2}$, Mengyao $\mathrm{Lv}^{2}$, Linchang An², Hui Xu${ }^{2}$ \\ ${ }^{1}$ China Meteorological Administration Public Meteorological Service Center, Beijing, China \\ ${ }^{2}$ National Meteorological Center, Beijing, China \\ Email: wuying_nmc@sina.com
}

How to cite this paper: $\mathrm{Wu}, \mathrm{Y} ., \mathrm{Lv}, \mathrm{M} . \mathrm{Y}$., An, L.C. and Xu, H. (2017) Analysis of Meteorological Conditions for Fog and Haze in the Eastern and Central Regions of China during January, 2013. Atmospheric and Climate Sciences, 7, 577-587. https://doi.org/10.4236/acs.2017.74042

Received: August 3, 2017

Accepted: October 28, 2017

Published: October 31, 2017

Copyright $\odot 2017$ by authors and Scientific Research Publishing Inc. This work is licensed under the Creative Commons Attribution International License (CC BY 4.0).

http://creativecommons.org/licenses/by/4.0/ (c) (i) Open Access

\begin{abstract}
Based on the data of conventional meteorological observation, NCEP reanalysis data and atmospheric composition observation, a comprehensive analysis of the three kinds of persistent fog and haze in eastern China in January 2013 was carried out. The results show that the process of persistent fog and haze is in the background of static weather, and the zonal circulation in the middle and high latitudes is not conducive to the south of the cold air. In the eastern part of China, near-surface wind speed is low under the controlled of pressure field, which is conducive to the formation and maintenance of haze. The formation of inversion layer, the height of the mixed layer, the stratified structure of the upper dry layer, the ground wind speed and so on can represent the static stability of the atmosphere. In the actual forecast, fog and haze can be distinguished from the angle of relative humidity, PM2.5 concentration, diurnal variation characteristics, mixed layer height and energy structure, industrial structure and local and surrounding economic development level.
\end{abstract}

\section{Keywords}

Continuous Haze, Inversion Temperature, Mixing Layer Height

\section{Introduction}

In recent years, due to frequent haze, human beings have become increasingly aware that haze is one of the atmospheric and ecological problems that can not be ignored [1] [2] [3]. In recent years, a lot of research has been done on haze at home and abroad [4] [5] [6]. Taking the haze pollution process in January 2013 as an example, we analyzed the present situation, the formation mechanism and 
the source composition of the haze pollution in Beijing and Tianjin, and put forward the control way from the aspects of political wisdom, management innovation, scientific support and universal participation [7]. Zhang Hengde et al. analyzed the characteristics, properties and circulation background of the large-scale continuous fog formation process in East China from January 30 to February 10, 2009. Diagnosing the water vapor and power conditions in the process of the fog, and comparing the difference between the process and the haze process in the eastern and central regions of China in early January 2008 [8].

Any country's urban or regional economic and social development process will encounter with the natural environment changes how to coordinate the problem. With the rapid development of China's economy, the process of urbanization is accelerating, the rapid development of the city caused serious pollution, visibility generally decreased, heavy haze events occur frequently. The haze pollution problem has become the shackles of China's rapid economic and social development. It is imperative to solve the contradiction between economic development and changes in the atmospheric environment. The formation of haze of fine particles through the direct absorption and scattering of solar radiation affect the Earth's radiation balance, so that the atmosphere warming or cooling, which directly affect climate change; but also as a condensed nucleus to change the micro-physical characteristics of the cloud, affecting the cloud of dust and precipitation, and then indirectly affect the climate.

A large number of scientific research work laid the theoretical and technical basis for fog and haze forecast. But for a wide range of haze weather, there is still less comprehensive analysis from different perspectives.

In this paper, the formation mechanism of haze will be studied from several aspects, such as large-scale circulation situation, atmospheric temperature and humidity structure and physical characterization of atmospheric static stability. The aim and goal of the present study are to find out the focus of the forecast to provide a reference for the future of such weather awareness and forecast.

\section{Fog and Haze Weather Process Characteristics}

In January 2013, there were three large-scale persistent fog and haze weather in most of the eastern and central regions of China. These 3 times were 7 th to 16th days, 22th to 24th days, 27th to 31th days. We analyze this several haze process, found the following characteristics:

1) Days of fog and haze are too many. In January 2013, most of the eastern and central regions had a haze of 5 to 10 days which in central and southern China, such as Jiangsu and Anhui, Zhejiang, Hubei and other places to reach 10 to 20 days, in some areas reached more than 20 days.

2) Long duration, wide range. There are more than 20 provinces and regions appear persistent haze. For example: range of haze reached 222 million square kilometers in January 22, covering almost the entire eastern region. And more 
appear for more than 5 days of haze weather, including Jiangsu, Anhui, Hunan, Hubei, Guangdong and other places lasting more than 10 days.

3) Heavy pollution, PM2.5 high concentration. Persistent haze caused a serious drop in air quality. The monitoring showed a large concentration of PM2.5 concentration exceeded. To Shijiazhuang, for example, 3 times the process, PM2.5 concentrations are mostly in more than $250 \mu \mathrm{g} / \mathrm{m}^{3}$, continued serious pollution for several days.

\section{Analysis of the Cause of Fog and Haze}

\subsection{Characteristics of Persistent Haze Circulation}

A wide range of persistent fog and haze processes occur in stable weather conditions. The average height field analysis of $500 \mathrm{hPa}$ in January 2013 shows that the mid-high latitudes of the Eurasia continent are under the control of the zonal circulation and the cold air intensity is weak. China's eastern and central regions affected by the western air, there is no strong cold air. In most cases weak cold rapid eastward movement of situation with short-wave trough, and with the humidity field is the performance of "dry on and wet down", that is, low humidity in the high, near the formation of high humidity which is conducive to the formation and maintenance of near-surface inversion. High altitude cloud cover is less, resulting in night surface radiation cooling. This facilitates the maintenance of the inversion structure at the boundary layer, providing a stable atmospheric environment for the formation and maintenance of haze and air pollution. From the analysis of the average sea level pressure field in January, the pressure gradient is small and the surface wind speed is small under the control of the pressure field in the eastern and central regions of China. These meteorological conditions are conducive to the formation and maintenance of haze.

\subsection{Analysis of Continuous Fog and Haze Weather Stability}

\subsubsection{Inversion Layer}

Usually the temperature in the atmosphere troposphere is decreasing with height. However, under certain special conditions, such as air sinking, radiation cooling or near ground disturbance and other factors under the influence of high temperature is higher than the lower layer, this phenomenon is called inversion. A layer of gas in the phenomenon of inversion, we call it inversion layer. The results show that the near-surface inversion layer suppresses atmospheric convection and is conducive to the appearance and development of fog and haze. In January 2013, there were inversion temperatures during the ongoing haze. For example: 925 - $1000 \mathrm{hPa}$ temperature difference in most parts of the eastern part of China are positive (Figure 1), there is a large area of the existence of temperature at 08:00 12th, 08:00 23th, 08:00 30th. This situation is conducive to the accumulation of water vapor near the ground. Also makes the humidity increase, the visibility decreases the formation of fog. While the existence of inversion layer is not conducive to the proliferation of pollutants dilution, resulting in significant decline in air quality, haze. 

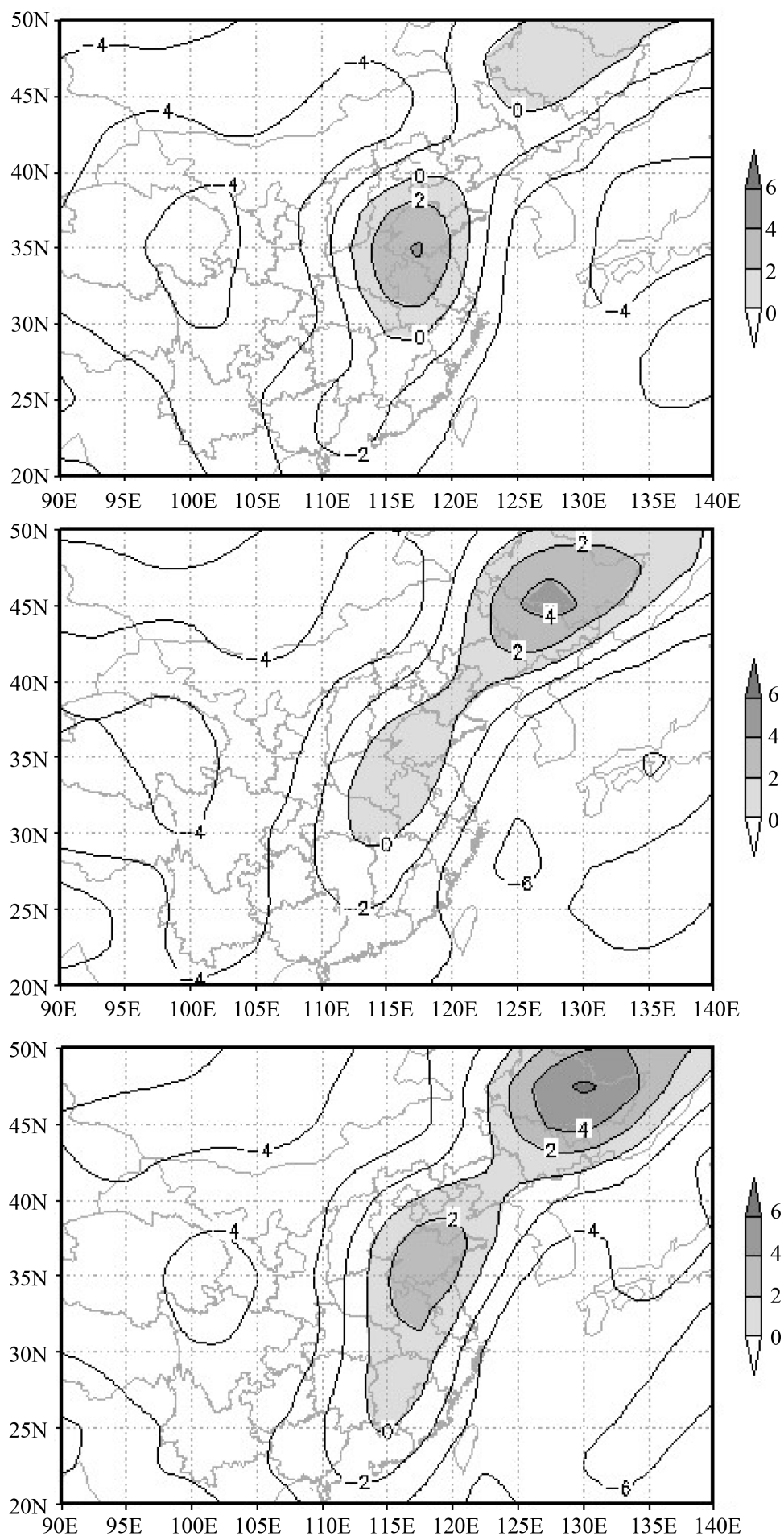

Figure 1. 925 - $1000 \mathrm{hPa}$ temperature difference at 08:00 12th (up) 08:00 23th (middle) 08:00 30th (below).

\subsubsection{Height of Mixing Layer}

If the turbulence of the lower air is strong, the upper turbulence is weak and there is a turbulent discontinuous interface in the middle, making it an impermeable or semipermeable reflective screen. The reflection screen reflects the 
bottom of the smoke back to suppress the lower air up delivery. Usually this discontinuous interface below the level of strong mixing be called mixed layer.

We believe that the atmospheric mixing layer is the result of a combination of thermal turbulence and mechanical turbulence. And there is mutual relation and feedback between the upper atmosphere and the ground meteorological parameters. The method of estimating the height of the atmosphere mixed with terrestrial meteorological data is called the Roche method [9]. We selected three times in the process of haze weather obvious time using the Roche method to calculate the height of the mixed layer. At 08:00 on January 13, 2013, the height of the mixed layer in most areas of the central and eastern regions was below $1 \mathrm{~km}$, local or even less than $0.4 \mathrm{~km}$. Corresponding ground observations in the eastern part of the visibility are less than $10 \mathrm{~km}$, there are different degrees of fog or haze. Corresponding to the height of the mixed layer is less than $0.4 \mathrm{~km}$ in Hunan, Guizhou, Jiangxi and other places, visibility is even less than $1 \mathrm{~km}$. At 08:00 and 20:00 on January 23,2013, the areas with low mixing layers are mainly concentrated in the north and south of the Yangtze River. At the same time ground observation data also show that this area is showing varying degrees of low visibility weather. The lower the height of the mixed layer, the more conducive to the accumulation of low-level water vapor, and promote the formation and development of fog. Contaminants in the vertical direction of the mixing is limited, easily lead to higher concentrations of pollution. It can be seen that the height of the mixed layer is clearly indicative of the forecast haze weather.

\subsection{Analysis of Water Vapor Conditions for Persistent Fog and Haze Weather}

The occurrence and development of fog and haze requires a certain amount of water vapor conditions, especially the formation of fog and the development of the ground near the relative humidity requirements. Taking the Beijing, Shijiazhuang and Nanjing stations as an example, the relative humidity vertical profile of 7 to 17 days (Figure 2) shows that the distribution of relative humidity during light fog to fog changes is also significant. Light fog corresponds to the ground relative humidity is generally $80 \%-90 \%$. While the visibility of the fog is less than $1 \mathrm{~km}$, the relative humidity is generally greater than $90 \%$. The relative humidity of haze is slightly smaller. It can be seen that the size of the relative humidity on the ground has a good indication of the generation and strengthening of the fog.

The strength of dry air is also closely related to the occurrence of fog. The presence of dry layers in the troposphere plays an important role in the occurrence and maintenance of fog. The wetting structure of the dry layer is conducive to the generation and development of fog. This is an important feature of foggy weather. As shown in Figure 2, Beijing, Shijiazhuang and Nanjing fog and haze weather are basically appear in the dry under the wet structure of the next layer. In general, a continuous fog process has several short trough activities, is conducive to the formation and maintenance of fog. Its effect is manifested in 

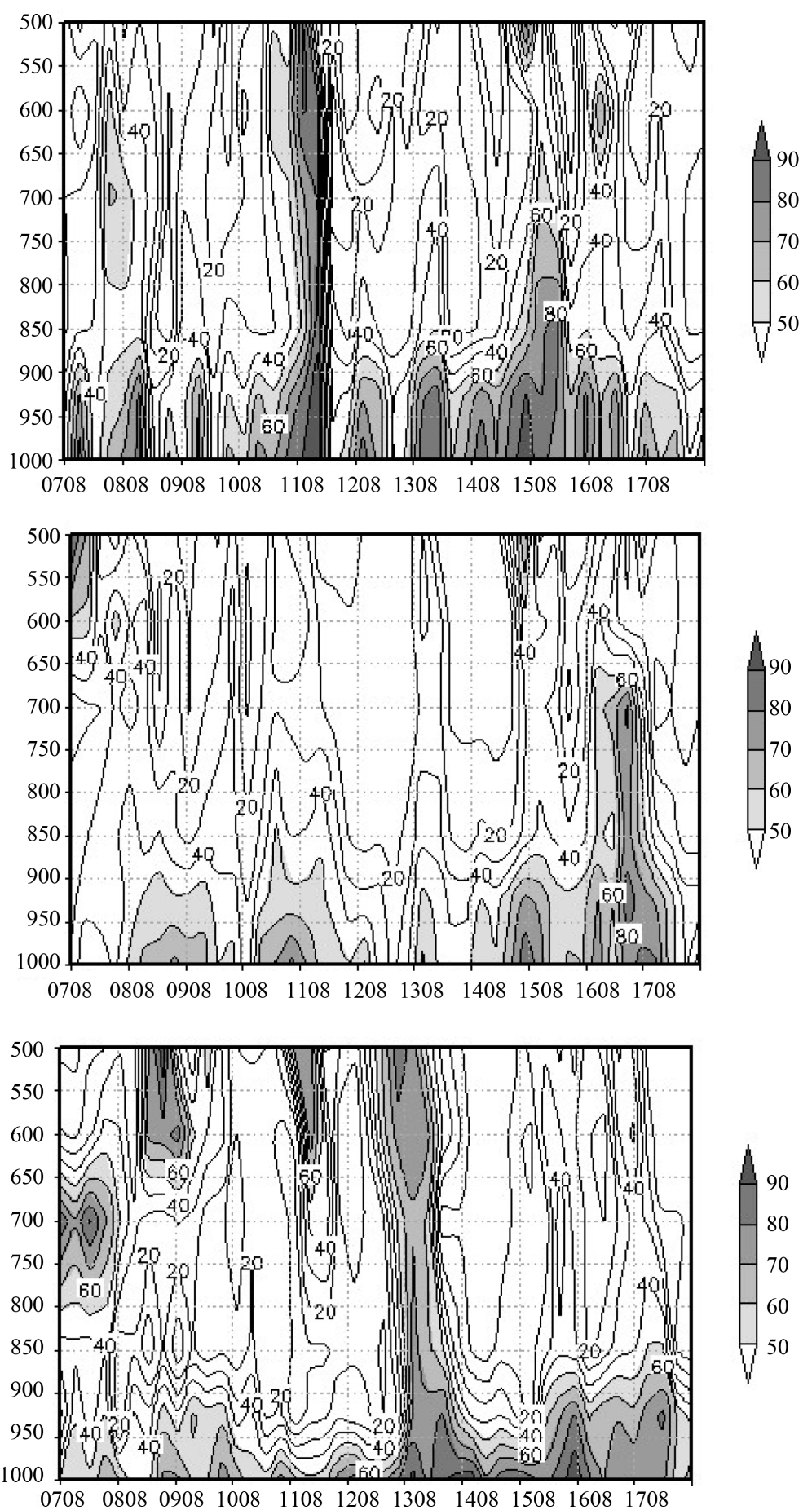

Figure 2. Time series chart of relative humidity vertical profile of Beijing (above), Shijiazhuang (middle), Nanjing (below) from 7th to 17th.

two aspects: on the one hand, the convection of the advection is favorable to the lower layer, and the existence of the middle and high layer is favorable to the radiation degradation of the stratum, which is easy to form the inversion temperature in the lower layer. On the other hand, the moisture flow is favorable for lower water vapor. 


\subsection{Analysis of Groundwater for Persistent Fog and Haze Weather}

Statistical haze observed in the process of visibility less than $1 \mathrm{~km}$ fog 4432 stations, haze 5916 times. The haze station of 10 meters $\mathrm{u}, \mathrm{v}$ wind speed most concentrated in the $0-2 \mathrm{~m} / \mathrm{s}$ between. This proves that the small wind speed on the ground is conducive to the appearance and maintenance of haze (Figure 3 ).

At the same time, it is found that the area of special terrain in the process of forecasting fog and haze should pay attention to the existence of ground convergence line. For example, in the eastern foot of the Taihang Mountains and Yanshan south, north of Hebei and southern Beijing in the terrain under the role of mesoscale convergence line. Under normal circumstances the west side of the convergence line for the northwest wind, east mostly east or southerly wind. For example, from January 11 to 13 , the surface wind field can be seen in the plain area east of the Taihang Mountains, in the plains east of the Taihang Mountains
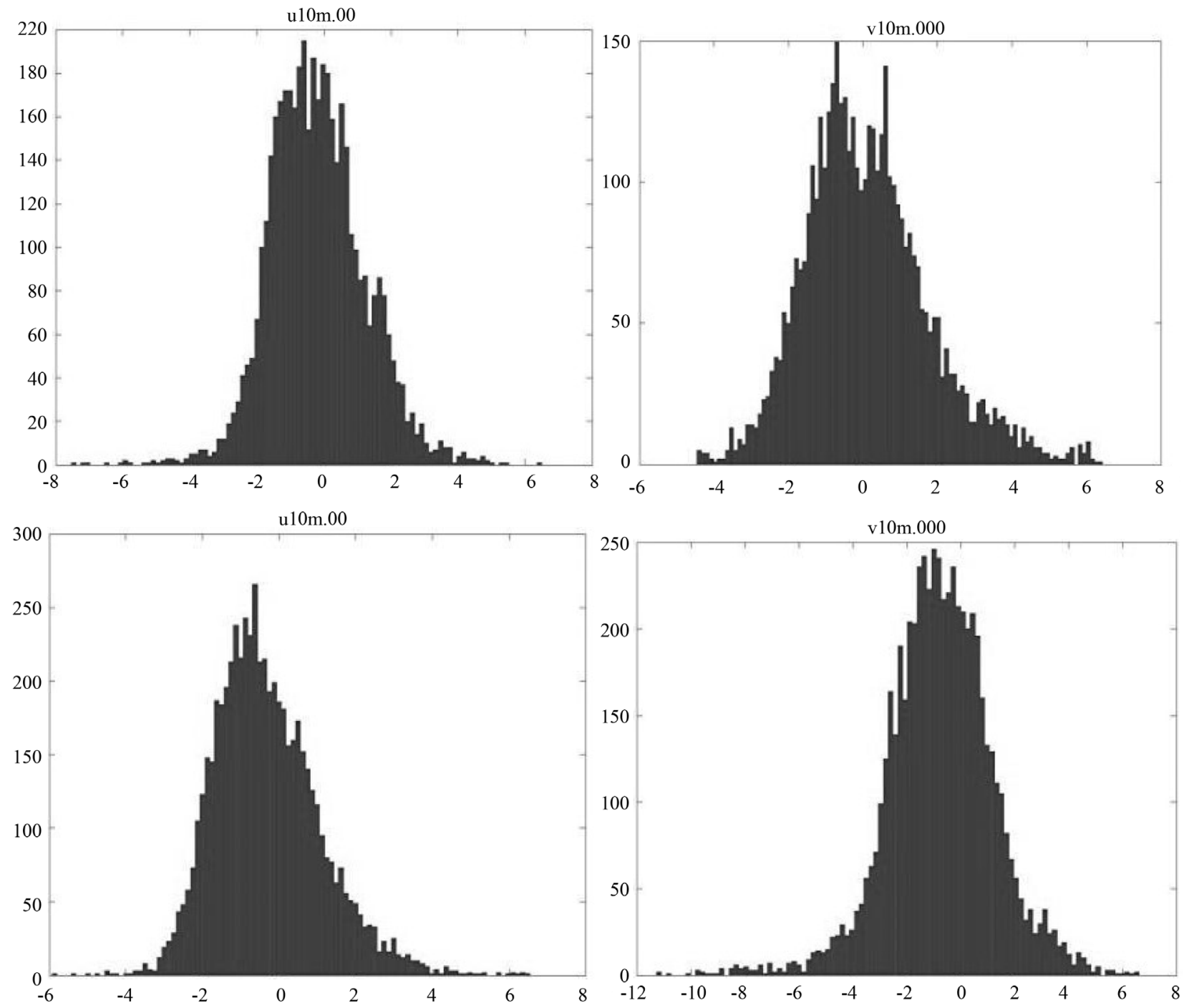

Figure 3. $10 \mathrm{~m} \mathrm{u}$ (above), v (below) wind speed (m/s) station distribution of fog (a) haze (b) weather. 
have emerged wind direction and wind speed convergence line, so that contaminants converge near the convergence line. And Shijiazhuang PM2.5 concentration curve during this time there have been significant peaks.

\section{The Correlation between Meteorological Elements and Visibility in Single Station}

\subsection{Analysis of Meteorological Factors and Visibility}

We selected two weather stations in Beijing and Nanjing to analyze the relationship between visibility and different meteorological factors (Figure 4). Beijing visibility is basically below $5 \mathrm{~km}$ in 10 to 14 days, Nanjing visibility is also less than $5 \mathrm{~km}$ in 7 to 16 days. First, from the wind speed point of view, low visibility during the wind speed is not large, more than $3 \mathrm{~m} / \mathrm{s}$, is not conducive to the proliferation of pollutants. Followed by the discovery of visibility and humidity
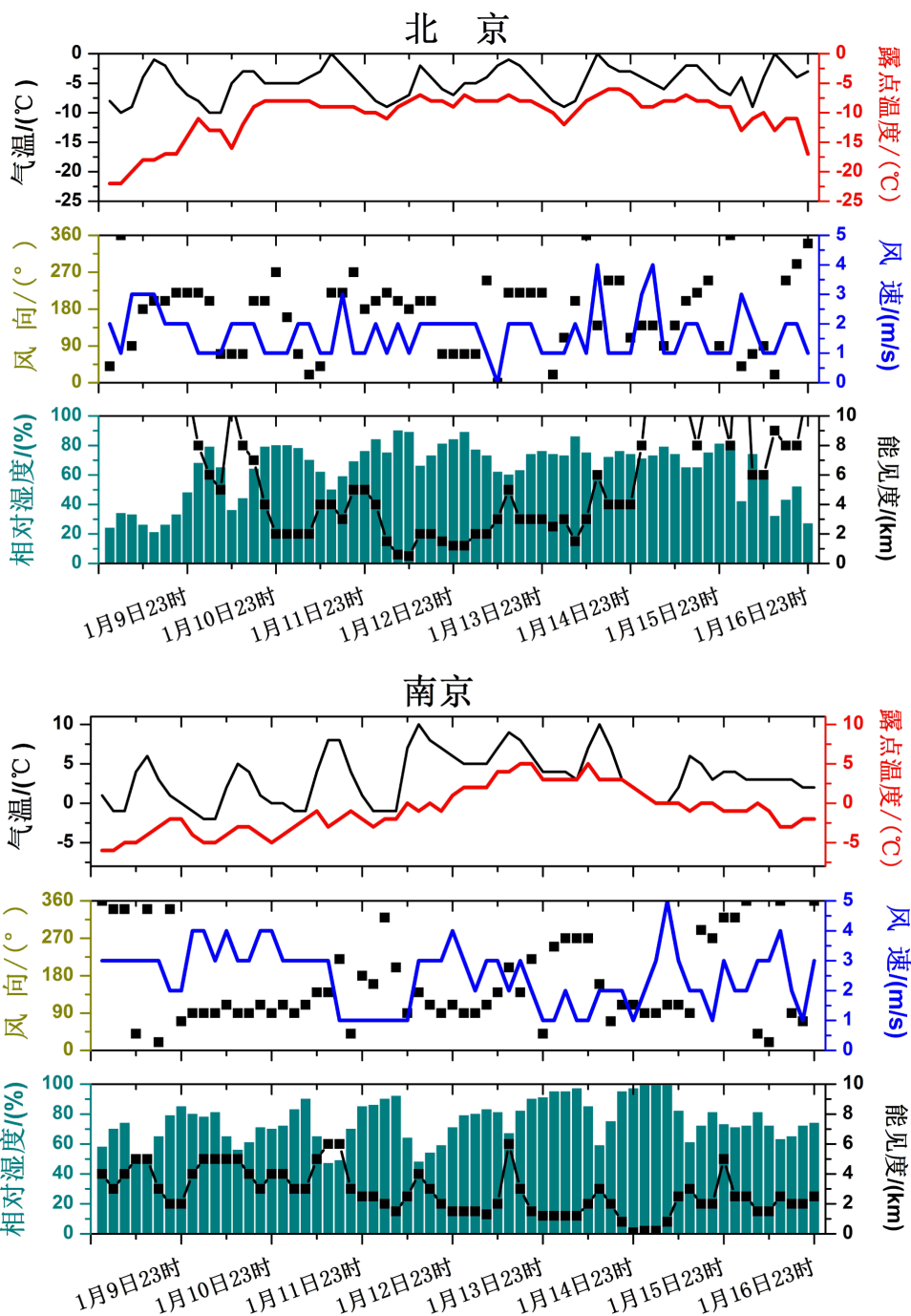

Figure 4. Beijing and Nanjing single station visibility and meteorological elements (temperature, dew point temperature, wind, relative humidity) changes from 7 th to 16 th. 
changes have a clear inverse correlation. There is a significant diurnal variation in relative humidity, high in the morning and lower in the afternoon. Corresponding to the visibility of the contrary, the morning low, significantly increased in the afternoon. The smaller the temperature dew point difference, the higher the air saturation, visibility will be reduced.

Beijing snow falls on the 10th night to $11 \mathrm{am}$. Visibility is not improved when snowfall occurs. Therefore, the cold air is weak, the atmosphere is stable, little wind speed, less precipitation, is not conducive to the proliferation and removal of pollutants. Beijing's fog appeared a clear strengthening stage on the morning of 12th. First, the atmospheric humidity conditions are stable, the temperature dew point difference is reduced, the relative humidity increases. Early heavy snowfall increased the ground humidity, while the nighttime radiation cooling is obvious, it is also conducive to the emergence of radiation fog. This is one of the causes of fog in Beijing where visibility is less than $1 \mathrm{~km}$. And Nanjing fog on the 14th night to strengthen the main reason is due to a sudden drop in ground temperature caused while the temperature drop is mainly the role of cold advection.

\subsection{Fog and Haze Mutual Conversion}

Nanjing station humidity less than $80 \%$ before 12 th, the air saturation is not enough, mostly haze-based, with the dew point temperature rising, the air vapor content continues to increase, the temperature dew point difference is reduced, is conducive to the formation of fog. The haze conversion mainly occurs during the period of 13th to 15th, mainly due to the rise in relative humidity due to the rise in temperature during the day and the dissipation of the fog. Since there was no significant cold air activity during the period, the atmosphere was stable and the contaminants accumulated in the near stratum were not diffused, resulting in a low level of visibility and the conversion of the weather into haze. After 14th, dew point temperature showed a downward trend, the air in the water vapor content decreased, and into the haze-based stage. It can be seen that the change in water vapor content in the air is the key to the haze conversion phase.

\section{Distinction between Fog and Haze}

As the formation of haze and fog atmospheric circulation background is basically similar, but there are differences in atmospheric composition, the two can be converted under certain conditions. So the fog and haze prediction ideas are basically similar, are to consider the atmosphere of the static situation. This makes it difficult to distinguish between fog and haze in the forecast process. We discuss the difference between fog and haze forecast below:

1) According to the standard of haze, the fog and haze can be distinguished from the relative humidity. The haze is considered when the air is near saturation and the haze is considered when the relative humidity is low. It is also possible to refer to the concentration of PM2.5 and the air quality index of the envi- 
ronmental observing station.

2) The fog may appear suddenly in a very short time, and the formation of haze is a gradual accumulation of results. So the visibility caused by haze will gradually increase.

3) According to statistics, haze appeared typical diurnal variation characteristics, the morning to morning fog frequency is higher, and after noon, the haze appeared frequency was significantly higher. You can distinguish fog and haze in different times of the forecast. Forecast haze, generally do not consider the seasonal differences, nor need to consider the sky situation.

4) Fog and haze appear mixed layer height is different from the fog when the height of the mixed layer is lower than the haze.

5) Haze and human activities are closely related to predict the emergence of haze, to consider the source of pollution conditions, haze not only with the local and surrounding areas of economic development, but also with the energy structure, industrial structure. For example, fossil fuels as the main energy structure, chemical, mining and other areas as the main industry, the haze is higher than the frequency of agriculture and animal husbandry-based areas.

\section{Conclusion}

Through the analysis of three large-scale persistent fog and haze weather in 2013, we draw the following conclusions: the persistence of a wide range of haze processes takes place in a stable weather background. In the high latitude in the zonal circulation situation, it is not conducive to cold air south. The pressure field control in China's central and eastern regions, near the surface wind speed is conducive to the formation and maintenance of haze. In January, 3 times the haze process are static weather generated. Inversion layer, the lower height of the mixed layer, and the dry layer under the wet structure of the atmosphere are to characterize the "stable". And the ground wind speed is small or even the emergence of static wind, reflecting the near formation of the "static". The haze and fog can be distinguished from the angle of relative humidity, PM2.5 concentration, diurnal variation characteristics, mixed layer height and pollution source condition.

\section{References}

[1] De, P.L., Acreman, S., Ashfold, M.J., Mohankumar, S.K. and Camposarceiz, A. (2015) The Link between Knowledge, Attitudes and Practices in Relation to Atmospheric Haze Pollution in Peninsular Malaysia. Plos One, 10, e0143655. https://doi.org/10.1371/journal.pone.0143655

[2] Ding, Y.H. and Liu, Y.J. (2014) Analysis of Long-Term Variations of Fog and Haze in China in Recent 50 Years and Their Relations with Atmospheric Humidity. Chinese Science: Earth Science, 57, 36-46.

[3] Chen, H. and Wang, H. (2015) Haze Days in North China and the Associated Atmospheric Circulations Based on Daily Visibility Data from 1960 to 2012. Journal of Geophysical Research Atmospheres, 120, 5895-5909.

https://doi.org/10.1002/2015JD023225 
[4] Gao, J., Woodward, A., Vardoulakis, S., Kovats, S., Wilkinson, P. and Li, L., et al. (2017) Haze, Public Health and Mitigation Measures in China: A Review of the Current Evidence for Further Policy Response. Science of the Total Environment, 578, 148.

[5] Zhang, M., Zhang, S., Feng, G., Su, H., Zhu, F. and Ren, M., et al. (2017) Indoor Airborne Particle Sources and Outdoor Haze Days Effect in Urban Office Areas in Guangzhou. Environmental Research, 154, 60.

[6] Wang, S., Yu, S., Li, P., Wang, L., Mehmood, K. and Liu, W., et al. (2017) A Study of Characteristics and Origins of Haze Pollution in Zhengzhou, China, Based on Observations and Hybrid Receptor Models. Aerosol \& Air Quality Research, 17, 513-528. https://doi.org/10.4209/aaqr.2016.06.0238

[7] Wang, Y.S., Yao, L., Liu, Z.R., et al. (2013) Beijing-Tianjin-Hebei Atmospheric Haze Pollution and Control Strategies. Chinese Academy of Sciences, 28, 353-354.

[8] Zhang, H.D., Rao, X.Q. and Qiao, L. (2011) A Large-Scale Continuous Fog Process Diagnosis in East China. Plateau Meteorology, 30, 1256-1258.

[9] Ma, F.J. (1990) A Method for Estimating the Height of Atmospheric Mixed Layers Using Conventional Meteorological Data. Environmental Science, 5, 11-14. 